\title{
Mechanical Thrombectomy for Stroke After Cardiac Surgery
}

\author{
Márcio Madeira, M.D.,* Catarina Martins, M.D., † Giovanna Koukoulis, M.D.,* \\ Marta Marques, M.D.,* João Reis, Ph.D., $\dagger$ and Miguel Abecassis, M.D.* \\ * Department of Cardiothoracic Surgery, Hospital de Santa Cruz, Carnaxide, Portugal; and \\ $\dagger$ †epartment of Neuroradiology, Centro Hospitalar Lisboa Central, Lisbon, Portugal
}

\begin{abstract}
Stroke after cardiac surgery remains a devastating complication and its treatment options are limited. Systemic fibrinolysis is a relative contraindication, because it raises the risk of systemic hemorrhage. Endovascular therapy, mechanical thrombectomy, and intra-arterial fibrinolysis have emerged as safer options. We present three patients who developed strokes following cardiac surgery who underwent successful mechanical thrombectomy and review the literature on this subject. doi: $10.1111 /$ jocs.12776 (J Card Surg 2016;31:517-520)
\end{abstract}

Stroke following cardiac surgery has a reported incidence of $1.7 \%$ to $2.5 \%$ following isolated coronary surgery, $1.8 \%$ to $3.6 \%$ following single valve replacement, $4.4 \%$ following combined coronary and valvular surgery, $6.7 \%$ to $7.4 \%$ following multivalve surgery, and $1 \%$ following congenital surgery. ${ }^{1-3}$ Treatment options are limited. Systemic fibrinolysis is contraindicated due to recent major surgery and it has increased bleeding risk. Alternatives are mechanical thrombectomy and intra-arterial fibrinolysis.

We report three cases of mechanical thrombectomy in patients with an acute ischemic stroke after cardiac surgery and review the literature on this technique.

\section{PATIENT PROFILES}

In our institution, stroke following cardiac surgery has a $2.1 \%$ incidence. During a two-year period there were 40 patients who developed a postoperative stroke of which three patients underwent mechanical thrombectomy.

The consent to publish this report was obtained from our institution's ethics commission.

\section{Patient 1}

A 72-year-old female with a history of hypertension and dyslipidemia underwent off-pump double coronary

Conflict of interest: The authors have no conflicts of interest. Funding: None.

Address for correspondence: Márcio F. Madeira, M.D., Department of Cardiothoracic Surgery, Hospital de Santa Cruz, Avenida Prof. Reinaldo dos Santos, 2790-134 Carnaxide, Portugal. Fax: 00351214188095; e-mail: madeira.marcio@gmail.com artery bypass grafting (CABG), with a left internal mammary artery to anterior descending artery and right internal mammary artery to the first obtuse marginal artery. There was no aortic manipulation. On postoperative day 1 (D1), after left atrium catheter removal, the patient developed right arm hemiparesis, dysarthria, right homonymous hemianopsia, and right cutaneous plantar reflex in extension.

The cranial computed tomography scan (CT) showed a left cortical-subcortical parietal and posterior temporal hypodensity, which were associated with an ischemic acute vascular injury in the left posterior cerebral artery (PCA) territory. The CT angiography revealed a filling defect on left $\mathrm{PCA}$ from the $\mathrm{P} 1$ segment (Fig. 1).

Angiography confirmed the left PCA occlusion from its proximal segment (Fig. 2A). Three hours after the onset of symptoms, mechanical thrombectomy using the TREVO ${ }^{\text {TM }}$ device (Streyker ${ }^{\text {TM }}$, Kalamazoo, MI, USA) was performed, with removal of an intraluminal thrombus and complete recanalization of the left PCA (Fig. 2B).

At discharge (D10), the patient recovered from most of her symptoms, with residual paresis of the right hand. The patient was medicated with acetylsalicylic acid (150 mg in day) plus clopidogrel (75 mg in day) for a year and only acetylsalicylic acid (150 mg in day) thereafter.

\section{Patient 2}

A 20-year-old female with severe pulmonary hypertension, who had undergone a left Blalock-Taussig shunt surgery at two years of age, underwent a right Blalock-Taussig shunt and atrial septostomy. On post- 


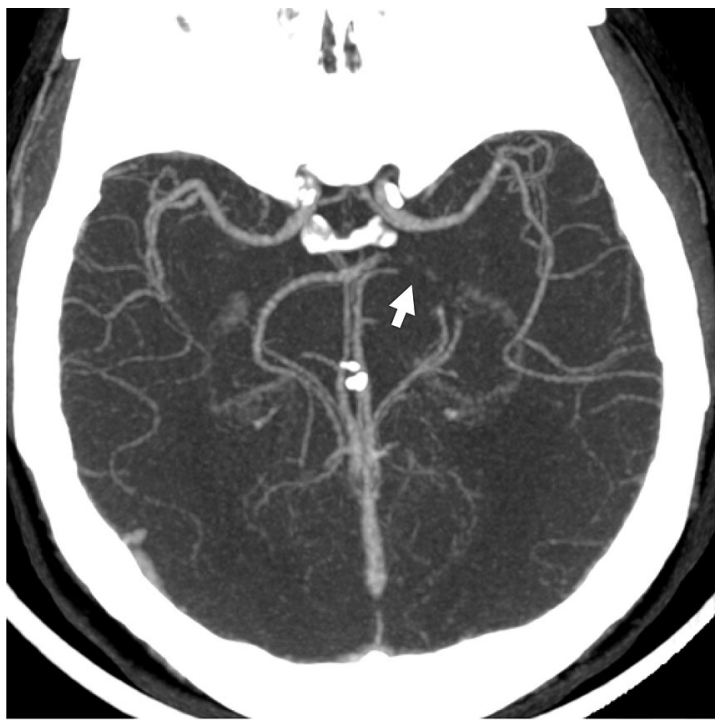

Figure 1. Cranial CT angiography: filling defect on P1 segment of left posterior cerebral artery (white arrow).

operative day 21 (D21), she was readmitted with a diagnosis of mitral valve endocarditis with a large sessile vegetation $(13 \times 8,6 \mathrm{~mm})$. On D24, she developed sudden left hemihypoesthesia, followed by left hemiparesis and dysarthria, which resolved spontaneously after some minutes. The CT showed no lesion; however, CT angiography demonstrated a nonocclusive thrombus at the right middle cerebral artery (MCA) bifurcation, in transition M1/M2 segments (Fig. 3).

Angiography confirmed the sub-total occlusion at the MCA bifurcation (Fig. 4A). Four hours after the onset of symptoms, mechanical thrombectomy was performed using the SOLITAIRE ${ }^{\text {TM }}$ device (manufactured by Covidien $^{\text {TM }}$, Minneapolis, MN, USA), with removal of an intraluminal thrombus with full recanalization $(\mathrm{TICl} 3)$ (Fig. 4B). The patient was anticoagulated with low

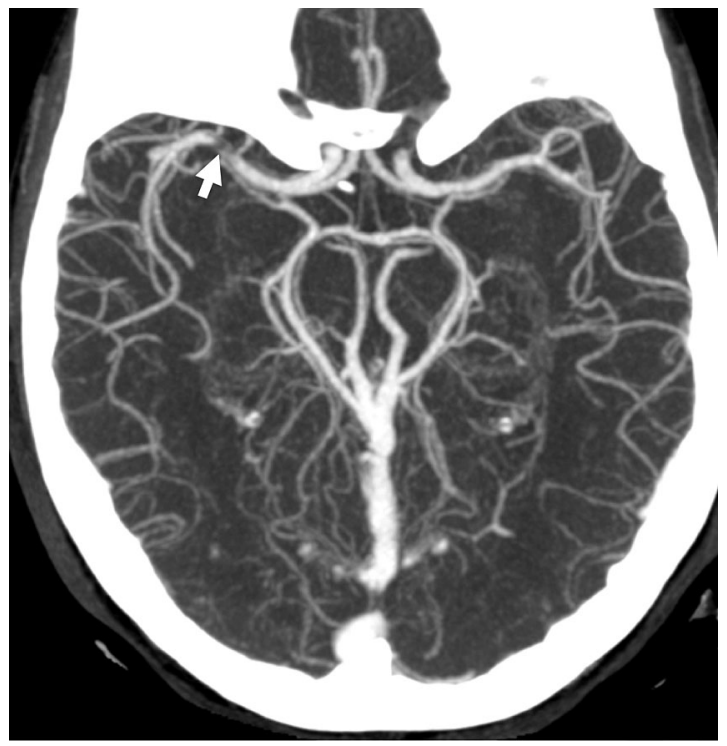

Figure 3. Cranial CT angiography: filling defect on the right middle cerebral artery bifurcation (white arrow).

molecular weight heparin for three days and after that she was medicated only with acetylsalicylic acid $(150 \mathrm{mg}$ in day). The full antibiotic regimen for endocarditis was concluded. At discharged she demonstrated complete neurological recovery.

\section{Patient 3}

Patient 3 was a 42-year-old female with a history of hypertension and hypothyroidism. She underwent a supracoronary aorta interposition graft for an ascending aortic aneurysm. On postoperative day 5 (D5) she suddenly developed mental status changes, anosognosia, right-sided head deviation, left homonymous hemianopsia, left central facial paresis, dysarthria, left hemiplegia, and left hemihypoesthesia. The CT showed early signs of ischemia in the territory of the right MCA.
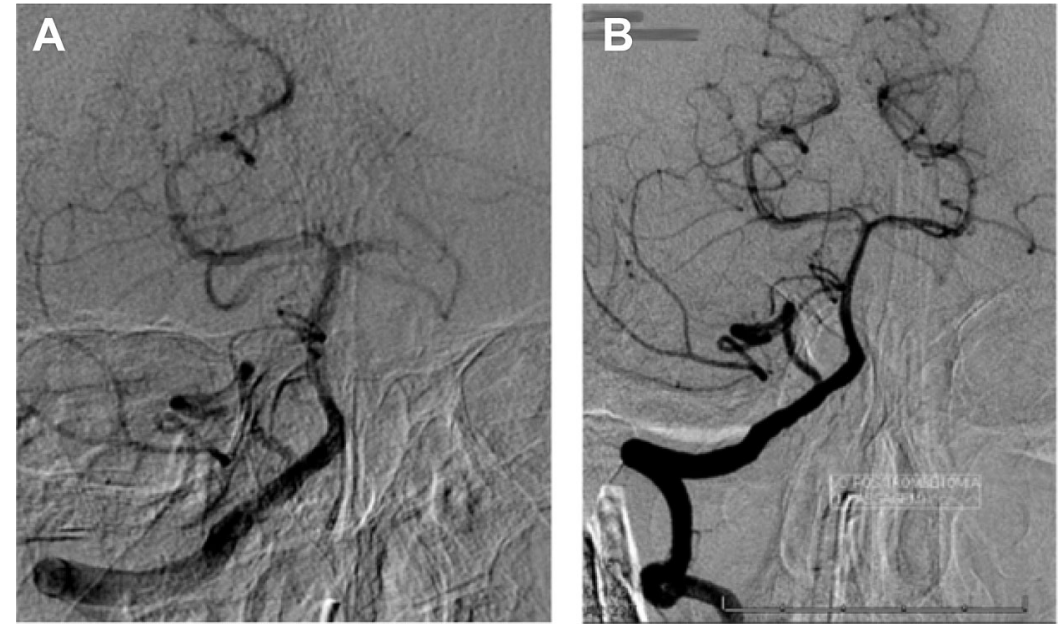

Figure 2. (A) Cerebral angiogram before thrombectomy: left posterior cerebral artery occlusion. (B) Cerebral angiogram after thrombectomy: total recanalization of left posterior cerebral artery. 

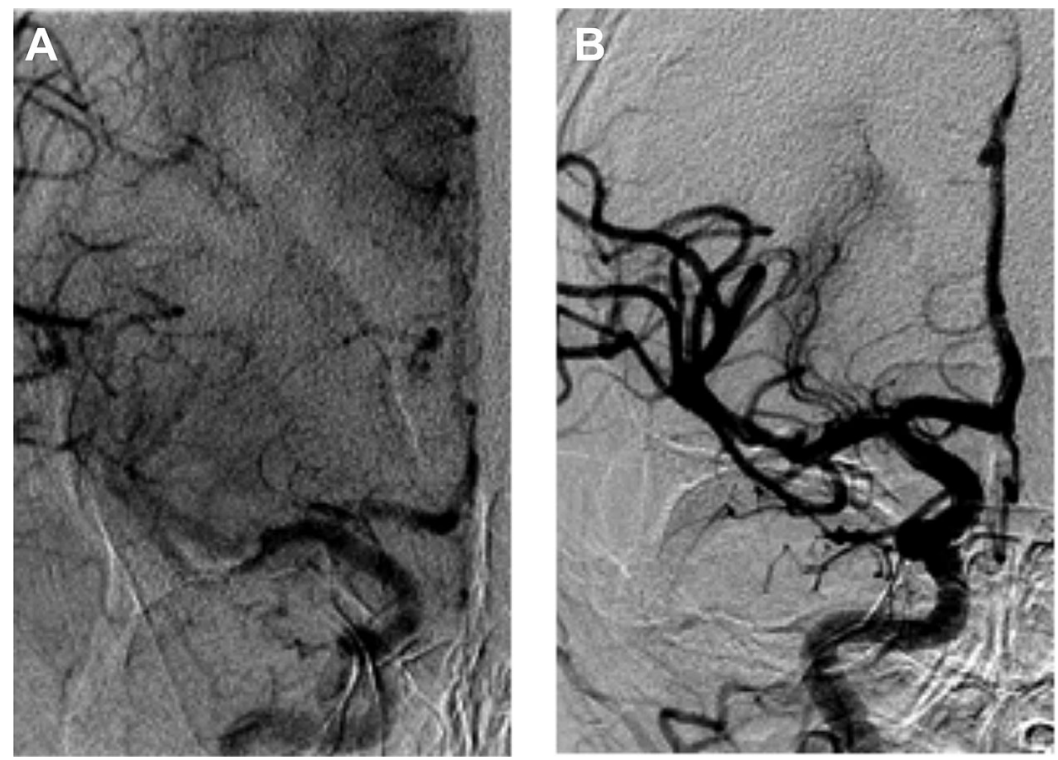

Figure 4. (A) Cerebral angiogram before thrombectomy: partial occlusion of the right middle cerebral artery. (B) Cerebral angiogram after thrombectomy: full recanalization of the right middle cerebral artery.

The CT angiography revealed occlusion of the proximal M1 segment of the right MCA (Fig. 5).

Angiography confirmed the occlusion of the M1 segment of the right MCA (Fig. 6A). Three and an half hours after the onset of symptoms, mechanical thrombectomy was performed with the TREVO ${ }^{\mathrm{TM}}$ device with complete removal of an intraluminal thrombus and full recanalization of the MCA circulation ( $\mathrm{TICl}$ 3) (Fig. 6B).

The patient recovered from her neurological defects while maintaining a slight left hemiparesis at discharge. The patient was anticoagulated with low molecular weight heparin during five days and after that with dabigatran (110 mg twice a day) for atrial fibrillation.

\section{DISCUSSION}

Despite improvements in ischemic stroke prevention measures, stroke after cardiac surgery has an incidence of $4 \%$ to $6 \%^{3}$ with high morbidity and mortality. One retrospective study revealed that mortality rates

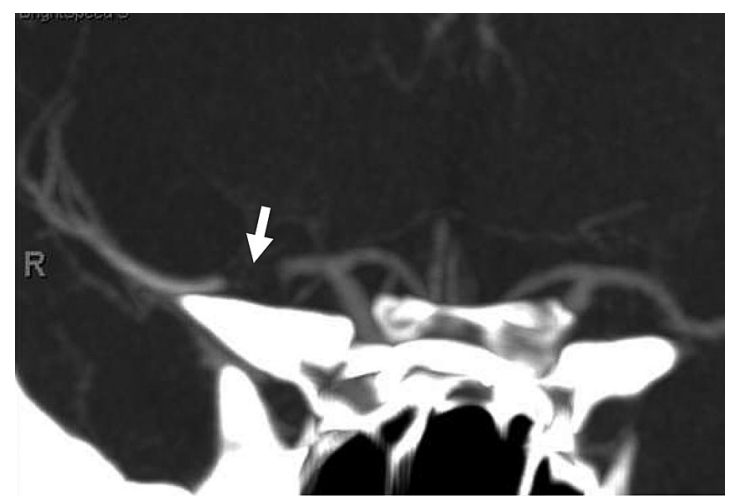

Figure 5. Cranial CT angiography: filling defect on the M1 segment of right middle cerebral artery (white arrow). one year after surgery in patients with stroke was $44 \%$, compared to $9 \%$ on patients without stroke. ${ }^{1}$

Given the contraindication to systemic fibrinolysis because of the high risk of hemorrhage, currently available alternative techniques are intra-arterial fibrinoIysis and mechanical thrombectomy. An important factor of mechanical thrombectomy is timing as it should be performed in the first six hours after the onset of symptoms. ${ }^{4}$

In the described cases, stroke occurred after extubation and recovery from sedation, so it was possible to identify the onset of symptoms, transfer the patient to an experienced center, and perform the specific procedure.

In the first two cases stroke etiology was probably embolic with total occlusion of a major intracranial artery. It was observed that in case 1 stroke occurred after catheter removal from the left atrium whereas in case 2 embolization was probably due to mitral valve vegetation.

$\mathrm{CT}$ and $\mathrm{CT}$ angiography demonstrated parenchymal lesions and the site of vessel occlusion. Cerebral angiographies were done minutes after the CT scans, and confirmed the specific cerebral vessel lesion.

In these cases immediate mechanical thrombectomy was an asset in acute stroke therapy. In the three patients, the thrombus was completely removed, with full recanalization of the vessel using TREVO ${ }^{\mathrm{TM}}$ devices in two and SOLITAIRE ${ }^{\mathrm{TM}}$ device in one patient. These two devices are both retrieval stents that are deployed within the thrombus, incorporating it and then removed. ${ }^{5}$ There are two other devices available: the Merci Retrievel System ${ }^{\text {TM }}$ (University of California, Los Angeles, CA, USA) that uses a memory shaped nitinol wire that is advanced in the thrombus to engage and remove it, and the aspiration devices that are designed to aspirate thrombus from larger vessels. ${ }^{5}$ Clinical trials support the superiority of retrieval stents compared to 

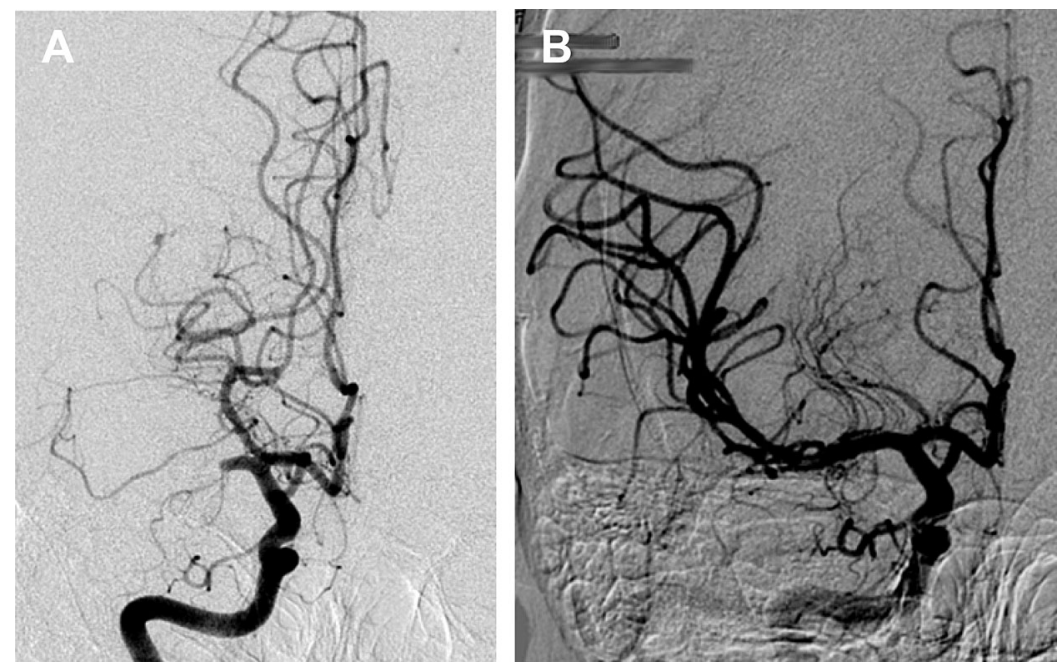

Figure 6. (A) Cerebral angiogram before thrombectomy: occlusion of the right $M 1$ segment of middle cerebral artery. (B) Cerebral angiogram after thrombectomy: full recanalization of the right middle cerebral artery.

Merci ${ }^{\mathrm{TM}}$ devices. ${ }^{5} \mathrm{~A}$ meta-analysis reported the efficacy and safety of mechanical thrombectomy in ischemic stroke. ${ }^{6}$ Mechanical thrombolysis had the highest recanalization rate $83.6 \%$ followed by intraarterial fibrinolysis with $63.3 \%$ and then intravenous fibrinolysis with $42.6 \% .{ }^{6}$ The 90 -day good neurological outcome rates (Modified Rankin Scale 0 to 2) for SOLITAIRE ${ }^{\text {TM }}$ and TREVO ${ }^{\text {TM }}$ devices were $51 \%$ and $47 \%$, respectively, with 90 day mortality of $14 \%$ and $31 \%$, respectively. ${ }^{7}$ The most frequent complications of this procedure are symptomatic intracranial hemorrhage, embolism to new territories, dissection, vasospasm, stent dislocation, or occlusion with reported cumulative rates of $11 \%{ }^{4}$

Mechanical thrombectomy is recommended as first line indication in large vessel occlusion if systemic thrombolysis is contraindicated. Mechanical thrombectomy should be performed at an experienced center and the decision to undertake the procedure should be made by a multidisciplinary team. The procedure may be contraindicated if there are signs of a large infarct. Although the time from the stroke to exclude patients from undergoing this technique has not been firmly established, the benefits are significantly reduced when reperfusion is undertaken after six hours. ${ }^{4}$

There are some studies that report the association of mechanical thrombectomy with intra-arterial fibrinolysis, but the major trials are made with mechanical thrombectomy alone. In mechanical thrombectomy with retrievable stents, antiplatelet or anticoagulant agents are not mandatory. ${ }^{5}$ For secondary prevention, antiplatelet agents are recommended and anticoagulation should be used in patients with cardioembolic stroke. 8
Acknowledgements: The authors thank Jaime Pamplona, M.D., Tiago Nolasco, M.D., Isabel Fragata, M.D., Tiago Baptista, M.D., and José Neves, M.D., for their contribution to the present work.

\section{REFERENCES}

1. Anyanwu AC, Filsoufi F, Salzberg SP, et al: Epidemiology of stroke after cardiac surgery in the current era. J Thorac Cardiovasc Surg 2007;134:1121-1127.

2. Boeken U, Litmathe J, Feindt $P$, et al: Neurological complications after cardiac surgery: Risk factors and correlation to the surgical procedure. Thorac Cardiovasc Surg 2005;53:33.

3. Bucerius J, Gummert JF, Borger MA, et al: Stroke after cardiac surgery: A risk factor analysis of 16,184 consecutive adult patients. Ann Thorac Surg 2003;75:472-478.

4. European Stroke Organisation, European Society for Minimally Invasive Neurological Therapy, European Society of Neuroradiology; Consensus statement on mechanical thrombectomy in acute ischemic stroke. Karolinska Stroke Update 2014.

5. Jauch EC, Saver JL, Adams HP, et al: Guidelines for the early management of patients with acute ischemic stroke. Stroke 2013:44:870-947.

6. Rha JH, Saver JL: The impact of recanalization on ischemic stroke outcome: A meta-analysis. Stroke 2007;38:967973.

7. Walcott BP, Boehm KM, Stapleton CJ, et al: Retrievable stent thrombectomy in the treatment of acute ischemic stroke: Analysis of a revolutionizing treatment technique. J Clin Neurosci 2013;20(10):1346-1349.

8. Kernan WN, Ovbiagele B, Black HR, et al: Guidelines for the prevention of stroke in patients with stroke and transient ischemic attack: A guideline for healthcare professionals from the American Heart Association/American Stroke Association. Stroke 2014;45(7):2160-236. 